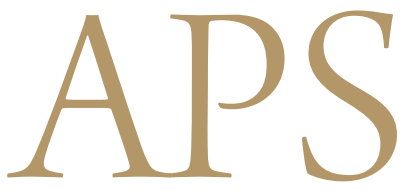

Archives of Plastic Surgery

\title{
Surgical Management of a Mandible Subcondylar Fracture
}

\author{
Dong Hee Kang \\ Department of Plastic Surgery, Dankook University Hospital, Dankook University College of Medicine, Cheonan, Korea
}

\begin{abstract}
Open reduction and anatomic reduction can create better function for the temporomandibular joint, compared with closed treatment in mandible fracture surgery. Therefore, the double miniplate fixation technique via mini-retromandibular incision was used in order to make the most stable fixation when performing subcondylar fracture surgery. Those approaches provide good visualization of the subcondyle from the posterior edge of the ramus, allow the surgeon to work perpendicularly to the fracture, and enable direct fracture management. Understanding the biomechanical load in the fixation of subcondylar fractures is also necessary in order to optimize fixation methods. Therefore, we measured the biomechanical loads of four different plate fixation techniques in the experimental model regarding mandibular subcondylar fractures. It was found that the loads measured in the two-plate fixation group with one dynamic compression plate (DCP) and one adaption plate showed the highest deformation and failure loads among the four fixation groups. The loads measured in the one DCP plate fixation group showed higher deformation and failure loads than the loads measured in the two adaption plate fixation group. Therefore, we conclude that the selection of the high profile plate (DCP) is also important in order to create a stable load in the subcondylar fracture.
\end{abstract}

Keywords Fracture fixation / Mandibular condyle / Mandibular fractures
Correspondence: Dong Hee Kang Department of Plastic Surgery, Dankook University Hospital, Dankook University College of Medicine, 359 Manghyangro, Dongnam-gu, Cheonan 330-715, Korea

Tel: $+82-41-550-6477$

Fax: +82-41-554-6477

E-mail:dhkcool@dankook.ac.kr

Received: 5 Apr $2012 \bullet$ Revised: 22 Jun $2012 \bullet$ Accepted: 22 Jun 2012

pISSN: 2234-6163 • elSSN: 2234-6171 • http://dx.doi.org/10.5999/aps.2012.39.4.284• Arch Plast Surg 2012;39:284-290

\section{INTRODUCTION}

The treatment for condylar neck and subcondylar fractures remains one of the most controversial topics of mandible surgery despite the high incidence of these fractures [1]. For decades, closed reduction has been the preferred treatment, but closed treatment requires varying periods of maxillomandibular fixation (MMF, 0 to 4 weeks) [2] and long term complications like pain, ankylosis, internal derangement of the temporomandibular joint (TMJ), as well as the inadequate restoration of the vertical height of the ramus, can possibly occur [2]. The debate continues over how to best manage subcondylar fractures and the question of which fractures should be treated surgically has yet to be answered [3]. However, in recent years, due to the enormous development of the osteosynthesis technique and the refinement of surgical techniques, the attitude towards the treatment of a condylar neck fracture has changed from an exclusively nonsurgical approach toward surgical treatment [4]. Recently, anatomic reduction and early mobilization of the jaw following surgery have been considered important for the functional rehabilitation of the TMJ [5]. When we operate on a subcondylar fracture, the treatment plan depends on, 1)

Copyright $\odot 2012$ The Korean Society of Plastic and Reconstructive Surgeons

This is an Open Access article distributed under the terms of the Creative Commons Attribution Non-Commercial License (http://creativecommons.org/

licenses/by-nc/3.0/) which permits unrestricted non-commercial use, distribution, and reproduction in any medium, provided the original work is properly cited.

www.e-aps.org 
whether open or closed reduction must be performed, 2) which approach to the fracture site will be used, and 3) what type of osteosynthesis is required.

\section{SURGICAL APPROACHES TO SUB- CONDYLAR FRACTURES}

During the application of surgical approaches, it is important to ensure that the surgeon is able to conduct anatomic reduction under direct vision of the completely exposed fractured end [4]. To treat subcondylar fractures, several surgical approaches have been reported and each approach has its own set of advantages and disadvantages. Therefore, the choice of surgical approach depends on the need to create an optimal view of the whole subcondyle without injury to the facial nerve or to the vascularization to the condylar head during surgery.

\section{Mini-retromandibular approach to low subcondylar fractures}

The retromandibular incision was first proposed by Hinds and Girotti [6] in 1967. It is favored by surgeons because it provides good visualization of the lower subcondyle and the entire ramus from the posterior border [7]. Compared with the submandibular incision, this incision is closer to the subcondyle; therefore, it provides direct access to the fractured line of the subcondyle and it enables straightforward fracture management, thus facilitating the reduction of the subcondyle. Furthermore, it allows the surgeon to work perpendicularly to the fracture so that excessive retraction can be avoided and use of a transfacial trochar is not necessary [1]. It can also avoid direct contact with the facial nerve and preserves the integrity of the parotid gland as well as its capsule and leaves a barely noticeable scar in a relatively hidden region [1]. The skin incision of this approach is located just posterior to the mandibular ramus and the most proximal point of the incision is just below the ear lobe, runs parallel down to the posterior border of the mandible, and is limited to $25 \mathrm{~mm}$ in length (Fig. 1). After the skin incision, the subcutaneous dissection allows for the extension of the surgical wound to the level of the fracture. After exposing the superficial musculoaponeurotic system (SMAS), a vertical incision is made through the SMAS behind the parotid gland. Blunt dissection is made through the parotid gland and masseteric fascia towards the posterior border of the mandible. This permits preservation of the facial nerve fibers, which are easily identified when they pass superficially to the masseter muscle and can be protected with a retractor [1]. After reaching the mandibular angle, the pterygomasseteric sling is thinned out until the bone surface becomes visible. A sharp cut is made through the periosteum at the posterior border of the ascending ramus, opening access to the whole ramus, which is dissected subperiosteally. The periosteum at the posterior border of the ramus is then incised, and subperiosteal dissection is continued to the condylar area until the fracture line and the displaced or dislocated proximal fragment are identified. The fragment is then repositioned under direct visualization of the fracture line. Anatomical adjustment can be facilitated by pulling the mandible downwards. After aligning the fragment, stable plate or screw osteosynthesis is carried out. The posterior border of the ramus and the mandibular notch serve as reference lines for correct three dimensional repositioning.

Wound closure is performed in layers after checking mandibular mobility and dental occlusion. A silastic drain is inserted close to the fracture line, with the exit location at the lower border of the incision line. The first step is refixation of the pterygomasseteric sling, followed by closure of the subcutaneous and cutaneous layers. The skin sutures are removed one week later. After the wound has fully healed, the scar is hardly visible.

\section{Fig. 1. Mini-retromandibular approach to low subcondylar fractures}

(A) Preoperative design of a mini-retromandibular incision. The proximal point of the incision is just below the ear lobe, runs parallel down to the posterior border of the mandible, and is limited to $25 \mathrm{~mm}$ in length. (B) It provides good visualization of the lower subcondyle and allows straightforward fracture management. (C) Postoperative X-ray, the two $2.0 \mathrm{~mm}$ adaption miniplates are applied to the anterior and posterior regions of the subcondyle. (D) The postoperative scar is hardly visible.



\section{Modified retromandibular approach to high subcondy- lar fractures}

There are some limitations with regard to retromandibular approaches, in that, if the subcondylar fractures are located in the high subcondyle and close to the condylar head, the space that can be exposed with this approach is restricted; therefore, we modify the retromandibular incision. The modified retromandibular approach provides the same exposure as the retromandibular and preauricular accesses combined, and it may be useful for procedures involving the high subcondylar region [8]. This approach provides a direct approach to the high subcondylar region and the distance from the skin incision to that region is reduced, compared to that of the routine retromandibular approach. The skin incision runs from the intertragal notch through the use of a gently curved incision around the ear lobule and uses the upper part of the retromandibular incision (Fig. 2). After transection of the skin, the skin flap is elevated, taking care not to injure the great auricular nerve, which lies over the sternocleidomastoid muscle, and the subcutaneous dissection is extended to the level of the fracture. After exposing the SMAS, a vertical incision is made through the SMAS onto the parotid gland extending from the ear lobe towards the gonial angle. As soon as the globular parotid tissue appears from the fascial incision, blunt dissection with a mosquito is used. When the trunk of the facial nerve or the temporofacial and cervicofacial division is exposed, the branches are retracted either superiorly or inferiorly depending on the location of the condylar fracture [7]. Once the posterior border of the mandible is reached, an incision is made through the pterygomasseteric sling. A periosteal elevator is used in order to strip the masseter muscle from the ramus and dissect superiorly to expose the fractured end along the posterior border of the condylar process. After fracture reduction and plating, the pterygomasseteric sling is reapproximated with sutures. The wound is also reapproximated in layers, and the SMAS is resuspended. Any violation of the parotid gland capsule must be closed tightly to prevent salivary fistula. A small drain placed into the subcutaneous space may be necessary to prevent a hematoma. The skin and subcutaneous tissue are then closed.

\section{FIXATION TECHNIQUES OF SUB- CONDYLAR FRACTURE}

In treating subcondylar fractures, osteosynthesis is still a functionally challenging problem. The subcondyle constitutes a relatively small bone that must withstand high compression and tensional force during the mouth opening exercise $[9,10]$. The introduction of metal micro- and miniplates and screws partially solves the problem of the stabilization of this fracture, in that it is now possible to stabilize the fracture with one or two plates in a more anatomic position than can be achieved with wires or with closed treatment $[2,4]$. Metal plates and fixation screws are continually being improved, and new forms and profiles of hardware are regularly developed and tested.

\section{Two-plate fixation technique}

The single-plate fixation technique does not provide sufficient strength to withstand the strains occurring in subcondylar fractures. Therefore, more and more authors advocate the use of a two-plate fixation technique, which seems to have the beneficial effect of restoring the tension and compression trajectories in subcondylar fractures. Ideally, two miniplates should be applied at the posterior and anterior border of the condylar neck in a triangular fashion with one plate below the sigmoid notch and another plate along the posterior border of the ramus [10]. There are several options for plate selection that can be used in subcondylar fractures, but we used the 2.0 mini-adaption plate and 2.0 dynamic compression plate (DCP) for reduction (Fig. 3).

\section{Fig. 2. Modified retromandibular approach to high subcondylar fractures}

(A) Preoperative design of modified retromandibular incision, black line. (B) It provides good visualization of the high subcondyle and allows straightforward fracture management. (C) Postoperative X-ray, the two $2.0 \mathrm{~mm}$ adaption miniplate are applied to the anterior and posterior regions of the subcondyle. (D) The postoperative scar is cosmetically acceptable.
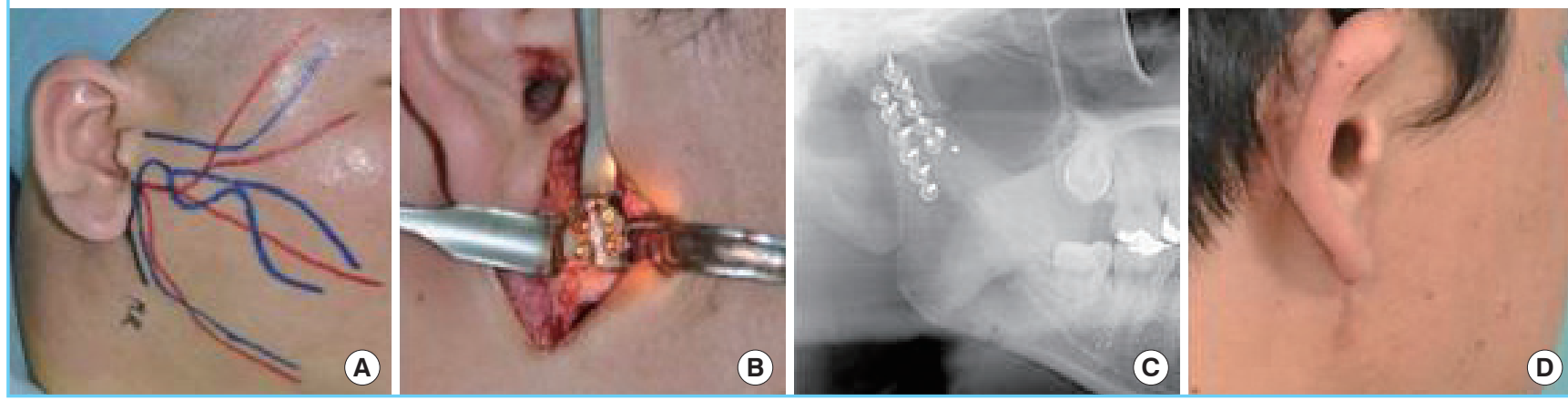


\section{Tests of two-plate fixation technique}

It remains uncertain whether plate fixation in condylar neck fractures is rigid enough to dispense with MMF and whether fixation techniques are strong enough to withstand functional loads encountered during the period of bone healing $[10,11]$. Although there is consensus that double miniplate fixation is the most stable fixation technique, there are few reports regarding which fixation technique provides functional stability in the fractures of this region $[7,10]$. Therefore, we measured the biomechanical load of four different two-plate fixation techniques in an experimental model of the mandibular subcondylar fracture [5]. Twenty standardized bovine tibia bones $(7 \times 1.5 \times 1.0$ $\mathrm{cm}$ ) were used for this study. Each of the four sets of tibia bones was cut to mimic a perpendicular subcondylar fracture in the

\section{Fig. 3. Two-plate fixation technique}

(A) Repeated tensile and compression forces are exerted on the subcondyle during mouth opening exercises. (B) The 2.0 miniadaption plate and the 2.0 dynamic compression plate were used for fracture reduction.
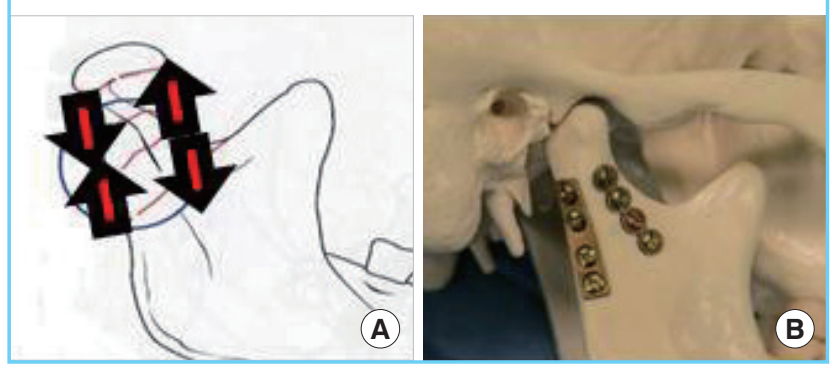

center area. The osteotomized tibia bone was fixed using one of the four different fixation groups (A-D). The fixation systems included the single $2.0 \mathrm{~mm} 4$ hole mini-adaption plate $(\mathrm{A})$, the single $2.0 \mathrm{~mm} 4$ hole DCP (B), double fixation with a $2.0 \mathrm{~mm}$ 4 hole mini-adaption plate (C), and double fixation with a 2.0 $\mathrm{mm} 4$ hole mini-adaption plate and a $2.0 \mathrm{~mm} 4$ hole DCP (D). A bending force was applied to the experimental model using a pressure machine (858 table top system, MTS, Minnesota, MN, USA) until failure occurred. The load for permanent deformation and maximum load of failure were measured in the load displacement curve with the chart recorder (Fig. 4).

The results of the analysis performed on our model are shown in Fig. 4 and Table 1. From the study, group D, to which was applied double fixation with a $2.0 \mathrm{~mm} 4$ hole mini-adaption plate and a $2.0 \mathrm{~mm} 4$ hole DCP showed the highest load for deformation and failure. Therefore, we can conclude that the use of a two-plate fixation method provides more a stable fixation load than single-plate fixation. In addition to that, the loads measured in the one DCP plate fixation group showed a higher deformation and failure load than the loads measured in the two adaption plate fixation group. Therefore, we conclude that the selection of the high profile plate (DCP) is also important in order to create a stable load when treating subcondylar fracture [5].

\section{Other plate uses in subcondylar fracture}

The two-plate technique seems to be a gold standard in subcon-

\section{Fig. 4. Fixation technique for subcondylar fractures with a $2.0 \mathrm{~mm}$ titanium miniplate and biomechanics model in each group}

(A) Group A. (B) Group B. (C) Group C. (D) Group D. (E) Measuring the load for permanent deformation and the maximum load for failure with an 858 table top system, MTS. (F) Load displacement curve in the MTS chart recorder. a, load for permanent deformation; $b$, maximum load for failure.
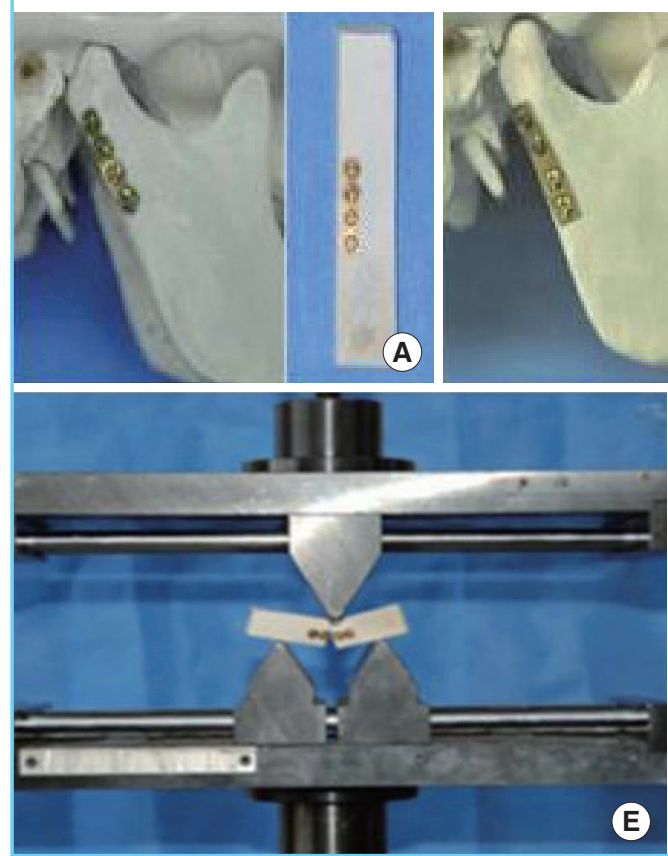
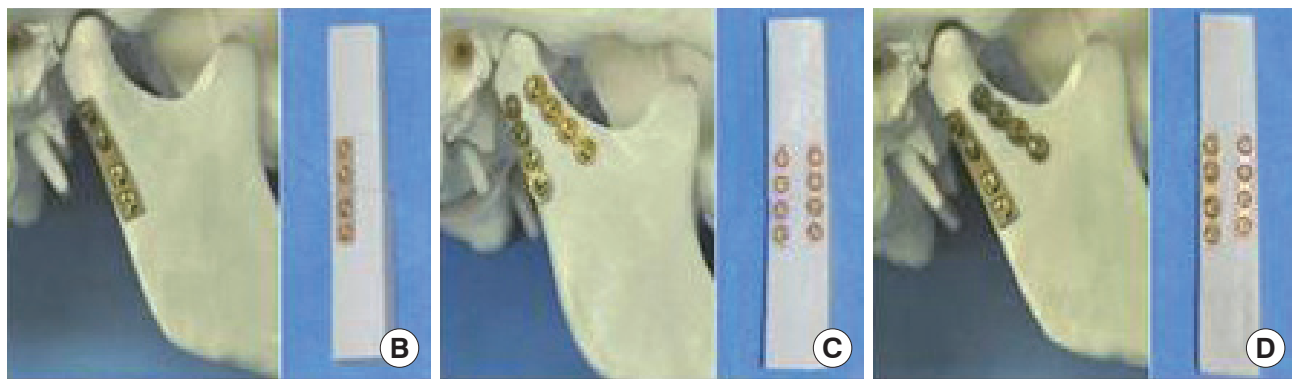

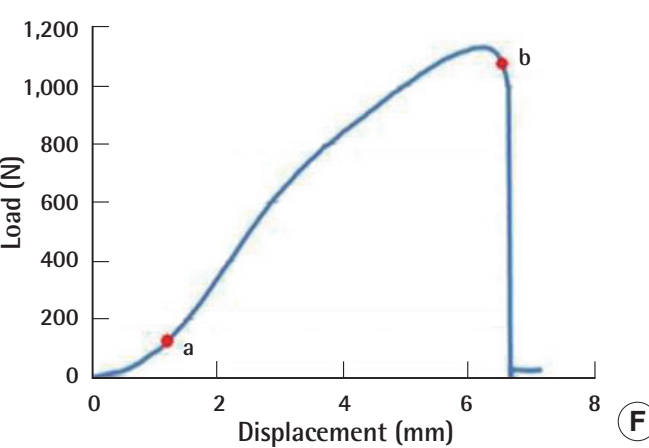




\begin{tabular}{|c|c|c|c|c|}
\hline Group & Type of stabilization & Number & $\begin{array}{l}\text { Load for permanent } \\
\text { deformation } \\
\text { (newton) }\end{array}$ & $\begin{array}{l}\text { Maximum load } \\
\text { for failure } \\
\text { (newton) }\end{array}$ \\
\hline A & Single adaption titanium miniplate 2.0 (four screw)a) & 5 & 121.7 & 450.0 \\
\hline B & Single dynamic compression titanium miniplate 2.0 (four screw) & 5 & 222.7 & 824.9 \\
\hline C & Two adaption titanium miniplate 2.0 (eight screw) & 5 & 174.3 & 693.9 \\
\hline \multirow[t]{2}{*}{ D } & Adaption titanium miniplate $2.0+$ dynamic compression titanium miniplate 2.0 (eight screw) & 5 & 247.3 & $1,223.6$ \\
\hline & Total & 20 & & $P<0.05^{a)}$ \\
\hline
\end{tabular}

\section{Fig. 5. Three kinds of plates were designed for the stabilization of subcondylar fractures}

(A) Trapezoid condyle plate plate. (B) Delta plate. (C) MODUS TriLock plate.
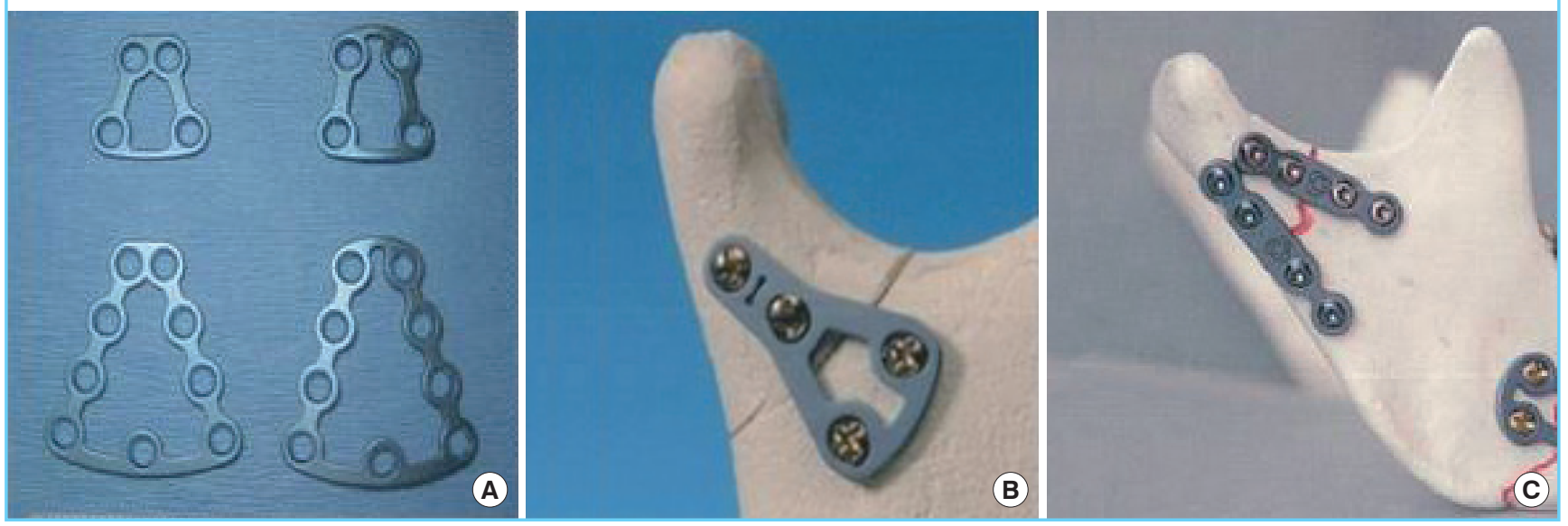

dylar fracture fixation but the insertion of many screws in a small condylar fragment is often difficult, particularly in the case of high subcondylar fractures. Miniaturized osteosynthesis devices are essential for stabilization of subcondylar fractures because of the small size of the subcondylar fragments [4]. Therefore, several kinds of plates have been specifically designed for the stabilization of subcondylar fractures, and they were subjected to rigorous experimental testing before clinical use [4].

The trapezoid shape of the TCP plate (Medartis, Basel, Switzerland) ensures the best possible stability of the fracture site and an optimal resistance of the plates to local mechanical strains (Fig. 5). The delta plate from Medartis is triangular in shape, oriented towards the angle of the mandible, and the lines of tensile and compressive stress run parallel to both sides of the triangle. The MODUS TriLock plate (Medartis) has been developed in accordance with a recent study regarding the lines of forces in the condylar region [4]. However, all of those plates made by Medartis are not yet available in the Republic of Korea.

\section{POSTOPERATIVE CARE}

The drain was removed within 3 to 4 days and the stitches removed within 7 days. MMF was not generally needed but restricted jaw movement with bandaging and a soft diet were used for 1 week. In a few patients who had malocclusion, elastic traction with a rubber band was used for several days. In most of the patients, limited mouth opening exercises were started several days after surgery and normal occlusion and mouth opening was restored within 1 month.

\section{DISCUSSION}

Treatment of subcondylar fractures can follow two different routes: conservative or surgical. Previously, conservative management of condylar fractures was favored. However, a open reduction was first applied to a low subcondylar fracture in 1925 [12], and recently it has become more common, probably because of the introduction of plate and screw fixation devices that allow for the stabilization of such injuries [1]. Today, many surgeons prefer open reduction of displaced fractures, because such reduction and rigid fixation enables good anatomic repositioning and immediate function. Although there is still debate concerning therapy for subcondylar fractures, a number of reports have now suggested that, compared with non-operative 
treatment, the treatment of condylar fractures by open reduction and rigid fixation creates more favorable results $[2,5]$.

The predominant surgical indication for adults is a dislocated or displaced condylar fracture outside the mandibular fossa, as it is generally impossible to attain anatomic reduction via conservative treatment [3]. According to the Zide and Kent criteria [3], the indications for open reduction were limited, because the techniques and materials available at that time were limited [4]. Over time, however, with the development of improved materials for fixation and the refinement of surgical techniques, the concept of rigid internal fixation has been increasingly applied to the injured craniomaxillofacial skeleton. Therefore, new considerations regarding the indications and advantages of open reduction have evolved. Today, for dislocated subcondylar fractures, open approaches are considered the treatment of choice in many surgical units.

The intended aim of surgical treatment regarding the condylar neck fracture is to restore the pre-existing anatomic relationships and acceptable function by means of stable osteosynthesis. The stability of osteosynthesis is influenced by the mechanical strains arising in the condylar region during mastication due to the action of the muscles acting on the mandible. A biomechanical study has shown that tension and compression forces are developed on the mandibular condyle region during normal mouth opening exercises, and that these strains correlate with the action of the muscles involved in mastication $[13,14]$. The magnitudes and directions of the principal components of strain on the four faces of the condylar process were first determined by Throckmorton and Dechow [13] who performed an in vitro study of human mandibles in 1994. The results demonstrated that the highest levels of tensile strain occur on the anterior and lateral surfaces, and the highest compressive strain occurs on the posterior surface. In 2002, Meyer's photoelastic analysis [14] demonstrated the presence of compressive stress patterns along the posterior border of the ramus and tensile stress patterns parallel and inferior to the sigmoid notch. These studies imply a need for new concepts for the application of osteosynthesis plates at the posterior and anterior border of the condylar neck in order to restore tension and compression trajectories [8].

Though it is difficult to accurately assess treatment results for the different plating techniques, using an in vitro model, our experiment shows that two-plate double fixation with an adaption plate and the DCP fixation technique produced the highest load for deformation and failure [5]. This technique may be considered a useful means for fixation in order to reduce the postoperative internal maxillary fixation period and achieve early mobility of the jaw. Furthermore, our results showed that the loads measured in the one DCP plate fixation group were higher than the loads measured in the two-adaption plate fixation group. Therefore, we conclude that the selection of the high profile plate (DCP) is also important to create a stable load in subcondylar fractures. It has already been proposed by Ellis and Dean [15] that the plate used in the management of condylar neck fractures should be stronger and thicker than the adaptation miniplate.

Although surgical management has been attempted in the hope of obtaining better results, some problems have remained, including difficulty in accessing the fracture site and insufficient or failed reduction of the fracture. When reduction of the condylar fragment is unsatisfactory and the condyle is more rigidly fixed in a nonphysiologic position, the risk of postoperative remodeling and degenerative change is too high because of the increased functional loading [16-18]. The risk of facial nerve injury is the problem that needs to be overcome. Therefore, careful reconsideration between the treatment efficacy and overall patient comfort is needed when we develop a treatment plan for subcondylar fractures.

\section{SUMMMARY}

In our cases, we always use the functionally stable two-plate fixation technique via mini-retromandibular and modified retromandibular incision in subcondylar fractures. Open reduction can restore the anatomic position of the subcondyle, thus yielding better function of the TMJ compared to closed reduction. A follow-up study of open reduction and internal fixation showed better radiologic results with regard to the mandibular ramus height, resorption, and pathologic change to the condyle, as compared with closed reduction. Hence, we conclude that the use of two correctly positioned plates for the stabilization of subcondylar fractures is currently the best solution in order to provide stable osteosynthesis in subcondylar fractures.

\section{REFERENCES}

1. Lee HC, Kang DH, Koo SH, et al. Outcome of open reduction via retromandibular approach for mandibular subcondyle fracture. J Korean Soc Plast Reconstr Surg 2005;32: 739-43.

2. Jang JY, Kang DH. Comparison study of open reduction and closed reduction in treatment of mandibular subcondylar fractures. J Korean Cleft Palate-Craniofac Assoc 2008;9:51-4.

3. Zide MF, Kent JN. Indications for open reduction of mandibular condyle fractures. J Oral Maxillofac Surg 1983;41: 89-98.

4. Kleinheinz J, Meyer C. Fractures of the mandibular condyle: 
basic considerations and treatment. London: Quintessence Publishing Compan; 2009.

5. Lee W, Kang DH. Study of the plating methods in the experimental model of mandibular subcondyle fracture. J Korean Cleft Palate-Craniofac Assoc 2011;12:12-6.

6. Hinds EC, Girotti WJ. Vertical subcondylar osteotomy: a reappraisal. Oral Surg Oral Med Oral Pathol 1967;24:16470.

7. Cienfuegos R, Carl-Peter Cornelius CP, Ellis E 3rd. et al. Mandible: retromandibular approaches [Internet]. Switzerland: AO Foundation; 2011 [cited 2012 Jun 27]. Available from: http://www2.aofoundation.org/wps/portal/surgery.

8. Tang W, Gao C, Long J, et al. Application of modified retromandibular approach indirectly from the anterior edge of the parotid gland in the surgical treatment of condylar fracture. J Oral Maxillofac Surg 2009;67:552-8.

9. Perthes G. Uber frakturen und luxationsfrakturen des kieferkopfchens und ihre operative behandlung. Arch Klin Chir 1924;133:418-33.

10. Choi BH, Kim KN, Kim HJ, et al. Evaluation of condylar neck fracture plating techniques. J Craniomaxillofac Surg 1999;27:109-12.

11. Tominaga K, Habu M, Khanal A, et al. Biomechanical evaluation of different types of rigid internal fixation techniques for subcondylar fractures. J Oral Maxillofac Surg 2006;64:
1510-6.

12. Silverman SL. A new operation for displaced fractures at the neck of the mandibular condyle. Dental Cosmos 1925;67: 876-7.

13. Throckmorton GS, Dechow PC. In vitro strain measurements in the condylar process of the human mandible. Arch Oral Biol 1994;39:853-67.

14. Meyer C, Kahn JL, Boutemi P, et al. Photoelastic analysis of bone deformation in the region of the mandibular condyle during mastication. J Craniomaxillofac Surg 2002;30:160-9.

15. Ellis E 3rd, Dean J. Rigid fixation of mandibular condyle fractures. Oral Surg Oral Med Oral Pathol 1993;76:6-15.

16. Throckmorton GS, Ellis E 3rd, Hayasaki H. Masticatory motion after surgical or nonsurgical treatment for unilateral fractures of the mandibular condylar process. J Oral Maxillofac Surg 2004;62:127-38.

17. Meyer C, Serhir L, Boutemi P. Experimental evaluation of three osteosynthesis devices used for stabilizing condylar fractures of the mandible. J Craniomaxillofac Surg 2006;34: 173-81.

18. Eckelt U, Schneider M, Erasmus F, et al. Open versus closed treatment of fractures of the mandibular condylar process-a prospective randomized multi-centre study. J Craniomaxillofac Surg 2006;34:306-14. 